\title{
Discovery and investigation of a naturally evolved fractal-like protein complex Franziska Sendker ${ }^{1}$, Stefan Bohn ${ }^{2}$, Jan Schuller ${ }^{3}$, Georg Hochberg ${ }^{4}$, Thomas Heimerl ${ }^{5}$, Pascal Pfister ${ }^{6}$ \\ ${ }^{1}$ Max Planck Institute for Terrestrial Microbiology ${ }^{2} \mathrm{Helmholtz}$ Zentrum Munich, ${ }^{3}$ Zentrum für Synthetische Mikrobiologie, ${ }^{4}$ Max Planck Institute for terrestrial Microbiology, ${ }^{5}$ Zentrum für Synthetische Mikrobiologie, ${ }^{6}$ Max Planck Institute for terrestrial Microbiology franziska.sendker@mpi-marburg.mpg.de
}

Many enzymes form homomeric complexes comprised of subunits encoded by the same gene. Assembly into homomeric complexes can build new active sites and enable particular types of allosteric regulation. Based on the many experimental structures of oligomeric proteins, sets of symmetry rules have been proposed and are thought to yield all conceivable geometries of homomers. Here we report the discovery of a citrate synthase that forms a wholly unprecedented kind of homomeric assembly not predicted by known symmetry rules. It closely resembles a famous deterministic fractal - the Sierpiński triangle. Molecular fractal assemblies have been a popular target in engineering efforts e.g. using small molecules that assemble on chiral surfaces, but have never been observed in nature or in solution. Using maximum likelihood phylogenetics, we show that this assembly evolved very recently from simpler dihedral hexamers within the cyanobacteria. To understand how this assembly is possible we have solved a cryo EM structure of the second fractal order: it relies on a symmetry violation within the hexamers that make up the fundamental building blocks of the Sierpiński triangle. Using mass photometry, we show that the assembly can be dissociated into hexamers in the presence of one of the enzyme's substrates, suggesting that it may represent a form of allosteric regulation. Our findings expand the space of known protein assemblies to selfterminating fractal-like complexes of different sizes. It further suggests engineering local symmetry violations into pre-existing dihedral complexes may yield totally new kinds of two-dimensional protein assemblies.

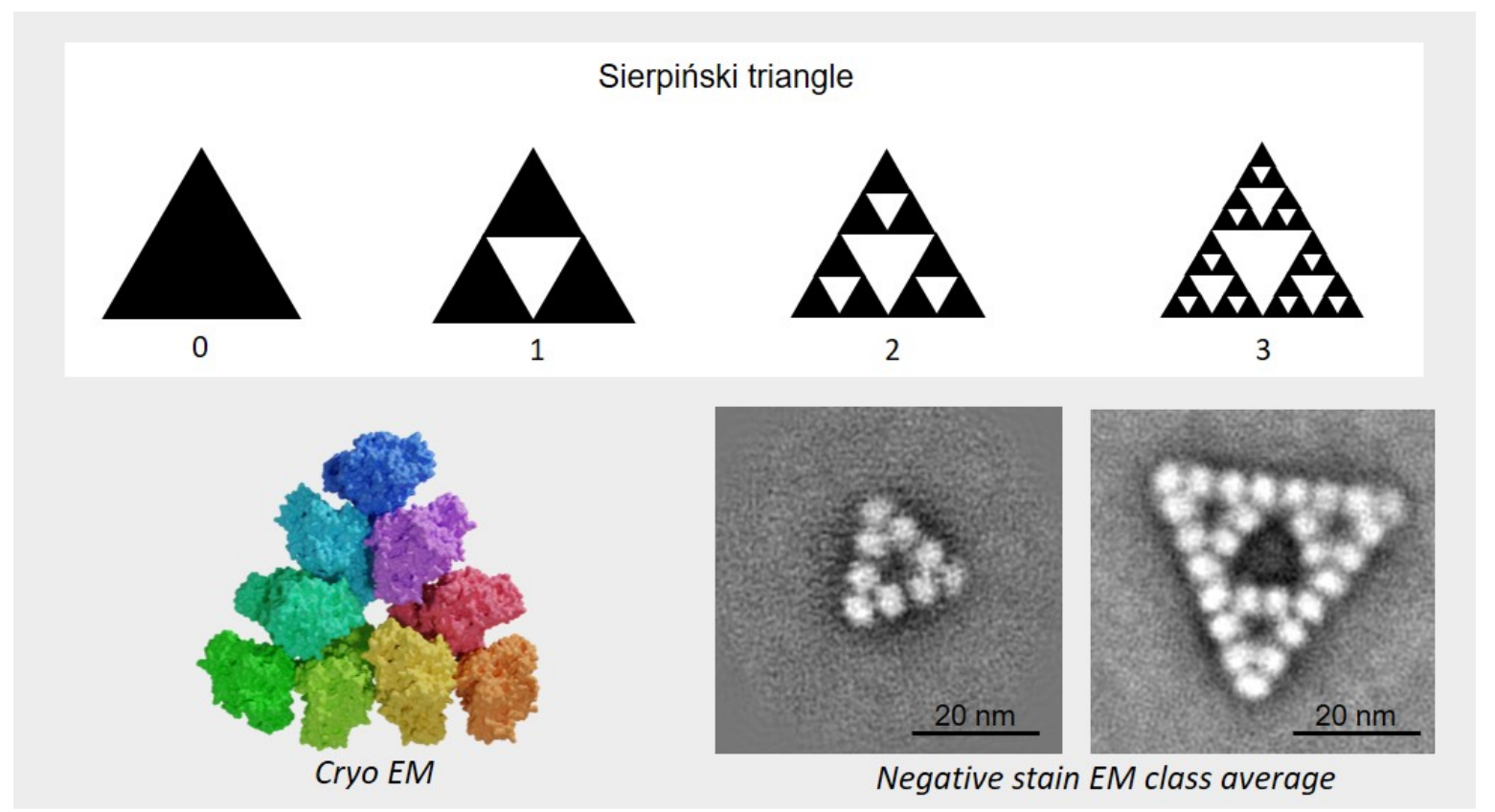

Figure 1 